\title{
Antifungal activities of essential oils and their constituents from indigenous cinnamon (Cinnamomum osmophloeum) leaves against wood decay fungi
}

\author{
Sheng-Yang Wang ${ }^{\mathrm{a}}$, Pin-Fun Chen ${ }^{\mathrm{b}}$, Shang-Tzen Chang ${ }^{\mathrm{b}, *}$ \\ ${ }^{a}$ Department of Forestry, National Chung-Hsing University, Taichung 402, Taiwan \\ ${ }^{\mathrm{b}}$ School of Forestry and Resource Conservation, National Taiwan University, No. 1, Section 4, Rossevelt Road, Taipei 106, Taiwan
}

Received 1 June 2003; received in revised form 12 July 2004; accepted 16 July 2004

Available online 18 September 2004

\begin{abstract}
Cinnamomum osmophloeum Kaneh is one of the hardwood species indigenous to Taiwan that possesses significant antifungal activity. To examine the antifungal activity of leaf essential oils and dominant constituents from C. osmophloeum, the essential oils of leaves from three clones (A, B, and C) collected from Haw-Lin experimental forest were extracted and their components analyzed by gas chromatography. Results from the antifungal tests demonstrated that the essential oils of both $\mathrm{B}$ and $\mathrm{C}$ leaves had strong inhibitory effects. The antifungal indices of these two leaf oils at $100 \mathrm{ppm}$ against five strains of white rot fungi and four strains of brown rot fungi were all $100 \%$. Cinnamaldehyde, the major compound in C. osmophloeum leaf essential oils, possessed the strongest antifungal activities compared with the other components. Its antifungal indices against both Coriolus versicolor and Laetiporus sulphureus were $100 \%$. The MIC (minimum inhibitory concentration) of cinnamaldehyde against C. versicolor and L. sulphureus was 50 and $75 \mathrm{ppm}$, respectively. In addition, comparisons of the antifungal indices of cinnamaldehyde's congeners proved that cinnamaldehyde exhibited the strongest antifungal activities.
\end{abstract}

(C) 2004 Elsevier Ltd. All rights reserved.

Keywords: Cinnamomum osmophloeum; Leaf; Essential oil; Cinnamaldehyde; White rot fungi; Brown rot fungi; Antifungal activity

\section{Introduction}

People have relied on trees for shelter, building materials, flavors/fragrances, and not least, medicines, since ancient times. The special properties of wood, including its aesthetic appearance, low density, low thermal expansion, and desirable mechanical strength, have led to indoor and outdoor applications. Durability is one of the most important considerations for the use of wood in construction. Poor durability has often been recognized as one of wood's "disadvantages" in certain

\footnotetext{
${ }^{*}$ Corresponding author. Tel.: +8862363 0231/3196; fax: +8862 3654520 .

E-mail address: peter@ntu.edu.tw (S.-T. Chang).
}

application. Developing methods to prolong the service life of wood has always been the interest of wood industry researchers. From the environmental perspective, finding naturally existent constituents in highly durable tree species and understanding their mechanisms are the most appropriate approaches to achieve wood protection while preserving the environment (Chang et al., 2000).

Owing to the unique ecosystem, there are many valuable tree species endemic to Taiwan. In the past few years, we have consistently studied the relationship between the properties and chemical constituents of endemic trees in Taiwan. According to the results obtained from our previous screening assay of endemic wood durability against fungi, we have found that Cinnamomum osmophloeum Kaneh (Lauraceae), named as 
"Indigenous cinnamon tree", is one of the hardwood species that possesses significant antifungal activity. $C$. osmophloeum is an endemic tree that grows at middle elevations in Taiwan's natural hardwood forest (Liu et al., 1988). Chemical constituents of its leaf essential oils are similar to those of the famous Cinnamomum cassia bark oil (Chang et al., 2001), so the potential utilizations of $C$. osmophloeum leaves are worthy of exploration. Leaves may be a source of effective pesticidal compounds and may come to be regarded as an inexhaustible source of friendly pesticides having low human and environment toxicity and being easily biodegradable. Although there have been few studies that discuss the antimicrobial and antitermitic activities of C. osmophloeum (Chang et al., 2001; Chang and Cheng, 2002), to our knowledge there is no literature concerning the antifungal properties of $C$. osmophloeum leaves against wood decay fungi. In this study, we distilled the leaf oils from three C. osmophloeum trees. After gas chromatography analysis and antifungal assays, the chemical constituents and the antifungal performances of these essential oils were investigated. The results are reported here. In addition, the antifungal activity of cinnamaldehyde congeners was subsequently examined to help to understand the effect of chemical structure on the antifungal activity.

\section{Methods}

\subsection{Collection of C. osmophloeum leaves and their essential oils}

The leaves of three twenty-year-old C. osmophloeum clones, named as tree $\mathrm{A}$, tree $\mathrm{B}$, and tree $\mathrm{C}$, were collected in May 2000 from the Haw-Lin Experimental Forest located in Taipei county. The species was identified, and the voucher specimens (COA01, COB01, and COC03) were deposited at the laboratory of wood chemistry, School of Forestry and Resource Conservation, National Taiwan University. The fresh leaf oils of C. osmophloeum were obtained by using water distillation for $6 \mathrm{~h}$ and their constituents determined by gas chromatography.

\subsection{Gas chromatography (GC) analysis}

GC was performed using a Shimadzu model-14B equipped with an FID. The column (Carbowax column) used was $50 \mathrm{~m}$ long by $0.22 \mathrm{~mm}$ i.d. glass capillary coated with silica. GC was programmed from 60 to $220^{\circ} \mathrm{C}$ at $2{ }^{\circ} \mathrm{C} / \mathrm{min} . \mathrm{N}_{2}$ was the carrier gas at the flow rate of $30 \mathrm{~mL} / \mathrm{min}$. Identification of the major components of indigenous cinnamon leaf oils was confirmed by comparison with standards, as well as by spiking. The quantity of compounds was obtained by integrating the peak area of the chromatograms. References standards were obtained from Acros (Belgium).

\subsection{Congeners of cinnamaldehyde}

For the structure-activity relationship study of cinnamaldehyde's derivatives, the congeners of cinnamaldehyde, including 4-hydroxybenzaldehyde, cinnamic acid, cinnamyl alcohol, and 3-phenylpropionaldehyde, were purchased from Acros (Belgium).

\subsection{Fungal strains}

In this study, five white rot fungi (Coriolus versicolor, Lenzites betulina, Pycnoporus coccineus, Trichaptum abietinum, and Oligoporus lowei) and four brown rot fungi (Laetiporus sulphureus, Antrodia taxa, Fomitopsis pinicola, and Phaeolus schweinitzii) were used in the antifungal assay. All of these fungal strains were gifts provided by Dr. Tun-Tschu Chang (Taiwan Forestry Research Institute).

\subsection{Antifungal assays}

Antifungal assays were performed based on the methods used in our previous studies (Chang et al., 1999, 2000) with slight modifications. Briefly, $100 \mathrm{ppm}$ of essential oils or compounds was added to sterilize potato dextrose agar (PDA). After transferring the mycelium of fungi, the test plates were incubated at $27^{\circ} \mathrm{C}$. When the mycelium of fungi reached the edges of the control plate (without added essential oils or compounds), the antifungal index was calculated as follows: antifungal index $=(1-\mathrm{Da} / \mathrm{Db}) \times 100$, where $\mathrm{Da}$ : the diameter of growth zone in the test plate. $\mathrm{Db}$ : the diameter of growth zone in the control plate. Each experiment was performed three times, and the data were averaged.

\subsection{Statistical analyses}

The Scheffe method was used to evaluate differences in antifungal index in antifungal tests. Results with $P<0.05$ were considered statistically significant.

\section{Results and discussion}

\subsection{Yields and chemical compositions of essential oils from different clones}

The yields of leaf oils from the three C. osmophloeum trees $(\mathrm{A}, \mathrm{B}$, and $\mathrm{C})$ were $6.02,6.92$, and $7.93 \mathrm{~mL} / \mathrm{kg}$ of leaves, respectively. Table 1 shows the main components and their relative contents of leaf essential oils distilled from C. osmophloeum. The relative amounts of each component were determined by the GC analysis. Cinna- 
Table 1

Main components and their relative contents (\%) of Cinnamonmum osmophloeum leaf essential oils

\begin{tabular}{|c|c|c|c|c|}
\hline \multirow[t]{2}{*}{ Compounds } & \multirow[t]{2}{*}{$\mathrm{RT}^{\mathrm{a}}(\min )$} & \multicolumn{3}{|c|}{ Relative percentages (\%) } \\
\hline & & Tree A & Tree B & Tree $\mathrm{C}$ \\
\hline 1,8-Cineol & 13.8 & 11.32 & $-{ }^{\mathrm{b}}$ & - \\
\hline Benzaldehyde & 33.0 & 0.93 & 2.88 & 3.20 \\
\hline Linalool & 34.8 & 9.83 & 0.12 & 0.24 \\
\hline Borneol & 43.6 & 7.46 & - & - \\
\hline$\alpha$-Terpineol & 44.3 & 4.62 & - & - \\
\hline Neral & 47.0 & 12.82 & - & - \\
\hline Geranyl acetate & 47.8 & 6.03 & 3.01 & 3.88 \\
\hline Geraniol & 53.4 & 4.73 & 2.58 & 2.96 \\
\hline Cinnamaldehyde & 64.6 & 8.35 & 81.08 & 76.00 \\
\hline Eugenol & 69.6 & - & 0.95 & 1.05 \\
\hline Cinnamyl acetate & 71.6 & 9.04 & 1.11 & 0.51 \\
\hline Coumarin & 84.1 & - & 0.50 & 0.26 \\
\hline
\end{tabular}

${ }^{\mathrm{a}} \mathrm{RT}$ : retention time, the analysis conditions as described in Section 2.

b -: trace.

maldehyde is the major compound in the essential oils of both indigenous cinnamon tree B $(81.08 \%)$ and indigenous cinnamon tree $\mathrm{C}(76 \%)$. In contrast, no dominant compound in the essential oil of indigenous cinnamon tree A was isolated, the amounts of each compound detected are lower than $13 \%$ (Table 1). According to the results obtained by $\mathrm{Hu}$ et al. (1985), there are nine different types of C. osmophloeum as classified by their chemical constituents, namely cassia type, cinnamaldehyde type, coumarin type, linalool type, eugenol type, camphor type, terpineol-4ol type, linalool-terpineol, and mixed type, respectively. On the basis of this classification, indigenous cinnamon tree $\mathrm{A}$, which was analyzed in this study, belongs to the mixed type. On the other hand, indigenous cinnamon trees $\mathrm{B}$ and $\mathrm{C}$ belong to the cinnamaldehyde type.

\subsection{Antifungal activity of essential oils}

It has been proven that cinnamon oil has inhibitory effects against several microorganisms, such as meat spoilage organisms (Ouattara et al., 1997) and fungi involved in respiratory tract mycoses (Singh et al., 1995). To the best of our knowledge, there has not been a relevant study investigating the effectiveness of indigenous cinnamon leaf oil and its constituents against wood decay fungi. To evaluate the antifungal activity of $C$. osmophloeum leaf essential oils, at first we selected two typical fungi, Coriolus versicolor (C.v., a white rot fungus) and Laetiporus sulphureus (L.s., a brown rot fungus), as test strains. Fig. 1 shows the antifungal activities of the leaf essential oils of $C$. osmophloeum clones at concentration of $100 \mathrm{ppm}$. Both brown rot fungus (L.s.) and white rot fungus (C.v.) were completely inhibited at doses of $100 \mathrm{ppm}$. Indigenous cinnamon trees $\mathrm{B}$ and $\mathrm{C}$ produced essential oils with much higher antifungal activities than those possessed by indigenous

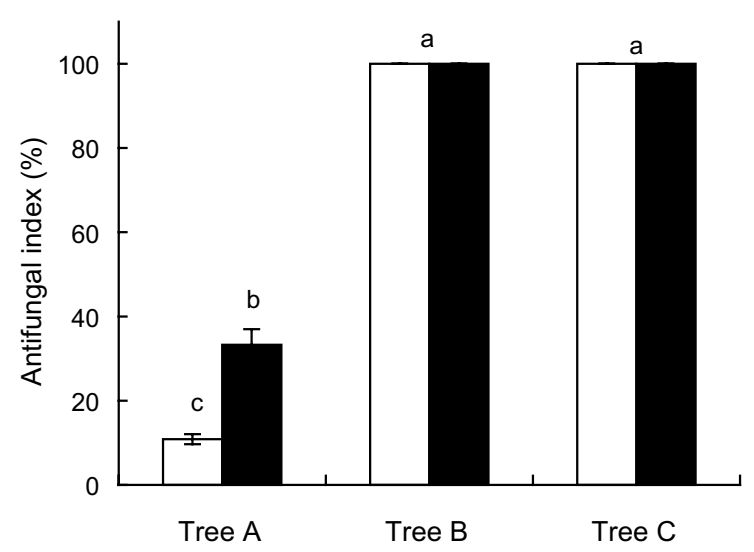

Fig. 1. Antifungal activity of three indigenous cinnamon leaf oils $(100 \mathrm{ppm})$ against the white rot fungus Coriolus versicolor and the brown rot fungus Laetiporus sulphureus. White bar: C. versicolor; black bar: L. sulphureus. Each experiment was performed three times, and the data averaged $(n=3)$. Numbers followed by different letters $(\mathrm{a}-\mathrm{c})$ are significantly different at the level of $P<0.05$ according to the Scheffe test.

cinnamon tree $\mathrm{A}$. The antifungal indices of indigenous cinnamon tree A were $18.37 \%$ and $34.47 \%$ against C.v. and L.s., respectively. The main constituent of essential oils from indigenous cinnamon trees $\mathrm{B}$ and $\mathrm{C}$ is cinnamaldehyde (approximately 80\%), whereas there is only $8.35 \%$ cinnamaldehyde in the essential oils from indigenous cinnamon tree A. Singh et al. (2000) have proven that cinnamaldehyde is a strong antifungal agent against human pathogens. According to their results, cinnamaldehyde may be a potential lead compound for the development of antifungal drugs through the control $\beta$-(1,3)-glucan and chitin synthesis in yeasts and molds (Bang et al., 2000). Thus, we suspected that cinnamaldehyde was the principal antifungal agent of C. osmophloeum leaf oil. 


\subsection{Antifungal effects of the main constituents of oils}

Tests were carried out to discover which of the eight main constituents of C. osmophloeum leaf essential oils possessed antifungal activity. As shown in Fig. 2, cinnamaldehyde and eugenol exhibited the strongest activity against both C.v. and L.s. amongst the eight compounds tested. Both cinnamaldehyde and eugenol totally inhibited the growth of L.s. (antifungal index $=100 \%$ ) at $100 \mathrm{ppm}$. Concerning C.v., however, eugenol (antifungal index $=71.19 \%$ ) showed a lower activity than cinnamaldehyde (antifungal index $=100 \%$ ). The effectiveness of all these compounds in terms of antifungal activity against C.v. at $100 \mathrm{ppm}$ is ranked as cinnamaldehyde $(100 \%)>$ eugenol $(71.19 \%)>$ cinnamyl alcohol $(25.68 \%)>$ coumarin $(17.41 \%)>$ geraniol $(15.73 \%)>$ linalool $(13.69 \%)>$ benzaldehyde $(11.37 \%)>\alpha$-terpineol $(6 \%)$. On the other hand, the order of antifungal indices against L.s. is cinnamaldehyde $(100 \%)=$ eugenol $(100 \%)>$ cinnamyl alcohol $\quad(50.71 \%)>$ coumarin $(37.88 \%)>$ geraniol $(33.53 \%)>$ benzaldehyde $(28 \%)>$ linanool $(0 \%)=\alpha$-terpineol $(0 \%)$. Although both cinnamaldehyde and eugenol possess stronger antifungal activity, eugenol exists in only minor quantities in the leaf oils of trees B and C (less than 1\%). The results obtained in this study point to cinnamaldehyde being the principal antifungal constituent of C. osmophloeum.

Since cinnamaldehyde possessed significant antifungal activities against both white rot fungus (C.v.) and brown rot fungus (L.s.), to further examine its antifungal activity we selected another seven strains of wood

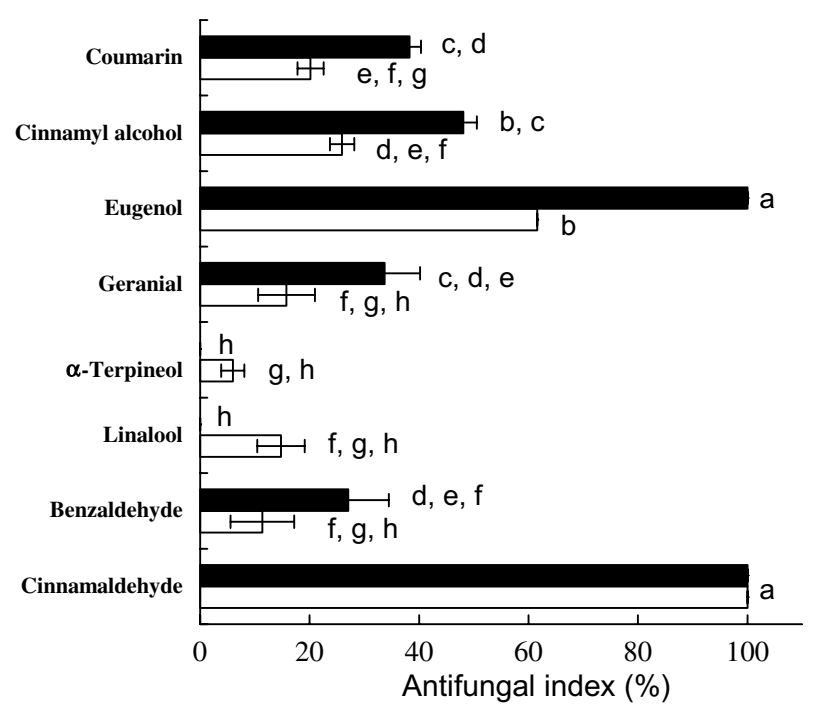

Fig. 2. Antifungal activity of the components (100 ppm) of indigenous cinnamon leaf oil against the white rot fungus Coriolus versicolor (white bar) and the brown rot fungus Laetiporus sulphureus (black bar). Each experiment was performed three times, and the data averaged $(n=3)$. Numbers followed by different letters $(\mathrm{a}-\mathrm{h})$ are significantly different at the level of $P<0.05$ according to the Scheffe test.
Table 2

Antifungal indices of cinnamaldehyde $(100 \mathrm{ppm})$ against wood rot fungi

\begin{tabular}{ll}
\hline Fungi & Antifungal index ${ }^{\mathrm{a}}(\%)$ \\
\hline White rot fungi & \\
Coriolus versicolor & 100 \\
Lenzites betulina & 100 \\
Pycnoporus coccineus & 100 \\
Trichaptum abietinum & 100 \\
Oligoporus lowei & 71.45 \\
Brown rot fungi & \\
Antrodia taxa & 100 \\
Fomitopsis pinicola & 100 \\
Laetiporus sulphureus & 100 \\
Phaeolus schweinitzii & 100 \\
\hline
\end{tabular}

${ }^{a}$ Antifungal index see Section 2. $100 \%=$ complete growth inhibition of mycelium.

decay fungi, which are commonly found in the forestry of Taiwan. Table 2 shows the antifungal indices of cinnamaldehyde against wood decay fungi at doses of $100 \mathrm{ppm}$. All of the selected fungi used in this study were totally inhibited by cinnamaldehyde except for Oligoporus lowei (white rot fungi category). The antifungal index of cinnamaldehyde was $71.45 \%$ against $O$. lowei. Over all the antifungal activity of cinnamaldehyde is excellent against wood decay fungi.

\subsection{Structure-activity relationship study of cinnamalde- hyde's derivatives}

According to the results obtained in this study, cinnamaldehyde is the strongest of antifungal compounds in the leaf oils of $C$. osmophloeum. To examine the structure-activity relationships of cinnamaldehyde, four compounds whose chemical structures are similar to cinnamaldehyde were selected for study. These four compounds were 4-hydroxybenzaldehyde, cinnamic acid, cinnamyl alcohol, and 3-phenylpropionaldehyde, and their chemical structures are shown in Fig. 3. Fig. 4 presents the results of antifungal activity of these compounds against both white rot fungus (C.v.) and brown rot fungus (L.S.) at doses of $100 \mathrm{ppm}$. It is obvious that cinnamaldehyde still possesses the strongest antifungal activity compared with the other leaf oil derivatives. When the antifungal activity of the cinnamaldehyde congeners having both a benzyl ring and a conjugated double bond was ranked: cinnamaldehyde $(100 \%$ and $100 \%$; antifungal activity against C.v. and L.s. $)>$ cinnamyl acid $(75.68 \%$ and $81.34 \%)>$ cinnamyl alcohol $(25.68 \%$ and $50.71 \%)>$ cinnamyl acetate (22.35\% and $34.24 \%)$. It is clear that compounds with an aldehyde group have the best antifungal activity. On the other hand, comparing with the compounds bearing with an aldehyde group, the antifungal indices 
<smiles>O=Cc1ccccc1</smiles>

1<smiles>O=Cc1ccc(O)cc1</smiles><smiles>OC/C=C/c1ccccc1</smiles>

3<smiles>O=C/C=C/c1ccccc1</smiles>

4

Fig. 3. Structures of cinnamaldehyde's congeners. (1) Benzaldehyde, (2) 4-hydroxybenzaldehyde, (3) cinnamyl alcohol, (4) cinnamaldehyde, (5) cinnamic acid, (6) cinnamyl acetate, and (7) 3 phenylpropionaldehyde.

of these categories were $100 \%, 69.56 \%, 12.47 \%$, and $11.73 \%$ for cinnamaldehyde, 3-phenylpropionaldehyde, 4-hydroxybenzaldehyde, and benzaldehyde, respectively. These results suggest that a compound having a conjugated double bond and a long $\mathrm{CH}$ chain outside the ring, i.e. cinnamaldehyde, possesses much stronger antifungal activity. A similar observation was noted in our previous study on the antitermitic activity of $C$. osmophloeum (Chang and Cheng, 2002). Amongst congeners of cinnamaldehyde, cinnamaldehyde exhibited the strongest termiticidal property. A similar finding was observed in other bioactivity assay. Motohashi et al. (2000) have evaluated the structure-activity rela- tionship in potential antitumor promoting benzalacetone derivatives, as assayed by the Epstein-Barr virus early antigen activation. Based upon their results obtained from EBV-EA activation inhibition assay model, the unsaturated bonded-carbonyl system is necessary for inhibition of mutagenesis activity in cinnamaldehyde derivatives.

\section{Conclusions}

To develop methods to prolong the service life of wood is one of the challenges of wood utilization researchers. With regard to environmental protection, finding "friendly preservatives" from highly durable tree species is an appropriate approach to achieve protection of the wood without polluting the environment. In this study, we investigated the antifungal activities of leaf essential oils from indigenous cinnamon tree (C. osmophloeum) against wood decay fungi, which has not been previously reported. Leaf essential oils of C. osmophloeum were proved to have strong effects on the growth inhibition of fungi. Cinnamaldehyde, the major compound in the leaf essential oils, possessed the strongest antifungal activities compared with the other components. Moreover, there are some special indigenous cinnamon tree clones (trees B and C), which contain high amount of cinnamaldehyde in the essential oils of leaves. That is to say, to obtain high yield of natural bioactive essential oils or cinnamaldehyde people could simply harvest the renewable leaves without logging or debarking the trees. We, therefore, suggest that the essential oils from indigenous cinnamon leaves may be further explored as a potential lead compound for the

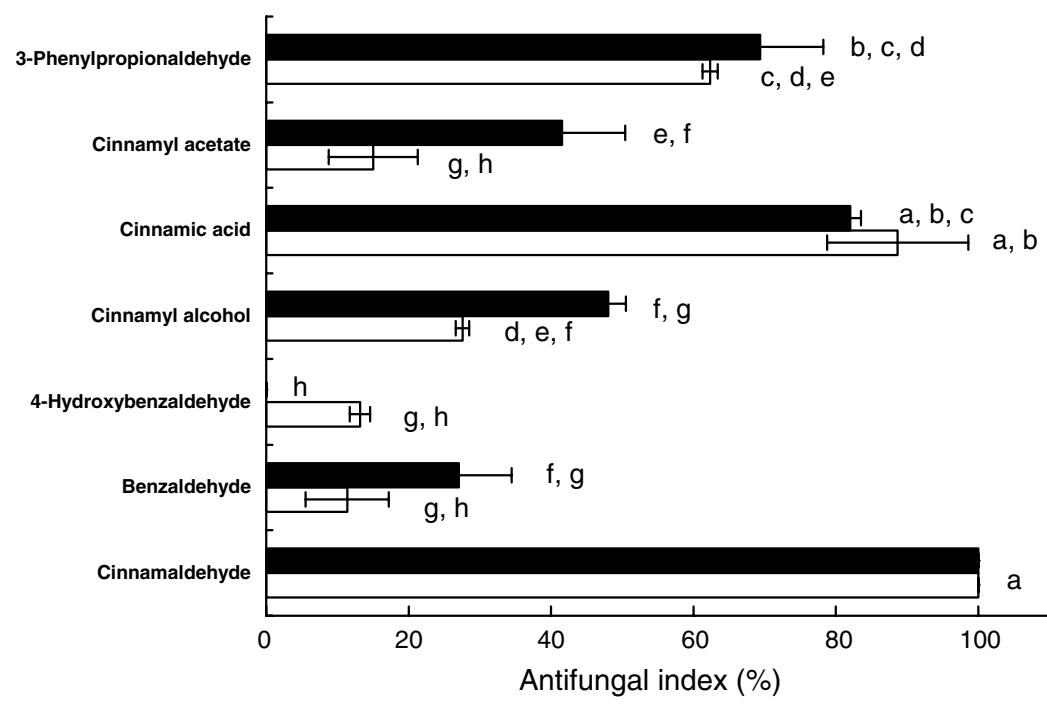

Fig. 4. Antifungal activity of the congeners of cinnamaldehyde at doses of $100 \mathrm{ppm}$. White bar: against $C$. versicolor; black bar: against $L$. sulphureus. Each experiment was performed three times, and the data averaged $(n=3)$. Numbers followed by different letters $(\mathrm{a}-\mathrm{h})$ are significantly different at the level of $P<0.05$ according to the Scheffe test. 
development of wood decay preservation agents or fumigants in the near future.

\section{References}

Bang, K.H., Lee, D.W., Park, H.M., Rhee, Y.H., 2000. Inhibition of fungal cell wall synthesizing enzymes by trans-cinnamaldehyde. Biosci. Biotech. Biochem. 64, 1061-1063.

Chang, S.T., Cheng, S.S., 2002. Antitermitic activity of leaf essential oils and components from Cinnamomum osmophloeum. J. Agric. Food Chem. 50, 1389-1392.

Chang, S.T., Chen, P.F., Chang, S.C., 2001. Antibacterial activity of leaf essential oils and their constituents from Cinnamomum osmophloeum. J. Ethnopharmacol. 77, 123-127.

Chang, S.T., Wang, S.Y., Wu, C.L., Su, Y.C, Kuo, Y.H., 1999. Antifungal compounds in the ethyl acetate soluble fraction of the extractives of Taiwania (Taiwania cryptomerioides Hayata) heartwood. Holzforschung 53, 487-490.

Chang, S.T., Wang, S.Y., Wu, C.L., Chen, P.F., Kuo, Y.H., 2000. Comparison of the antifungal activity of cadinane skeletal sesqui- terpenoids from Taiwania (Taiwania cryptomerioides Hayata) heartwood. Holzforschung 54, 241-245.

Hu, T.W., Lin, Y.T., Ho, C.K., 1985. Natural variation of chemical components of the leaf oil of Cinnamomum osmophloeum Kaneh. Bull. Taiwan Res. Inst. Eng. 78, 18.

Liu, Y.C., Lu, F.Y., Ou, C.H., 1988. Trees of Taiwan; Monographic Public no. 7, College of Agriculture, National Chung-Hshing University, Taichung, Taiwan, p. 136.

Motohashi, N., Yamagami, C., Tokuda, H., Okuda, Y., Ichiishi, E., Mukainaka, T., Nishino, H., Saito, Y., 2000. Structure-activity relationship in potentially anti-tumor promoting benzalacetone derivatives, as assayed by the Epstein-Barr virus early antigen activation. Mutat. Res.-Genet. Toxicol. Environ. Mutat. 464, $247-$ 254.

Ouattara, B., Simard, R.E., Holley, R.A., Piette, G.J.P., Begin, A., 1997. Antibacterial activity of selected fatty acids and essential oils against six meat spoilage organisms. Int. J. Food Microbiol. 37, $155-162$.

Singh, H.B., Srivastava, M., Singh, A.B., Srivastava, A.K., 1995. Cinnamon bark oil, a potent fungitoxicant against fungi causing respiratory tract mycoses. Allergy 50, 995-999. 